\title{
Lipidome Alterations Induced by Cystic Fibrosis, CFTR Mutation and Lung Function
}

Adriana Zardini Buzatto ${ }^{1}$, Mai Abdel Jabar ${ }^{2}$, Imran Nizami $^{3}$, Majed Dasouki ${ }^{2}$, Liang Li ${ }^{1 *}$, Anas

M. Abdel Rahman ${ }^{2,4,5^{*}}$

${ }^{1}$ Department of Chemistry, University of Alberta, Edmonton, AB, T6G 2R3, Canada

${ }^{2}$ Department of Genetics, King Faisal Specialist Hospital and Research Centre (KFSHRC),

Zahrawi Street, Al Maather, Riyadh 11211, Saudi Arabia

${ }^{3}$ Lung Transplant Section, Organ Transplant Center, King Faisal Specialist Hospital and

Research Center, Zahrawi Street, Al Maather, Riyadh 11211, Saudi Arabia

${ }^{4}$ Department of Biochemistry and Molecular Medicine' College of Medicine, Al Faisal

University, Riyadh, Saudi Arabia

${ }^{5}$ Department of Chemistry, Memorial University of Newfoundland, St. John's, NL, A1B 3X7,

Canada

\section{*Correspondence to:}

Anas Abdel Rahman, Ph.D.,

Department of Genetics, Research Center

King Faisal Specialist Hospital and Research

Centre (KFSHRC), Riyadh 11211, Kingdom of

Saudi Arabia

Email: aabdelrahman46@kfshrc.edu.sa

\section{Liang Li, PhD}

Department of Chemistry, University of Alberta, Edmonton, AB T6G 2R3, Canada

Email: liang.li@ualberta.ca 


\section{Table of Contents}

Supp. Table S-1. Patient cohort employed for this study. ( $x l s x)$

Supp. Table S-2. Composition of the deuterated internal standard mixture in methanol (Splash Lipidomix Mass Spec Standard, Avanti Polar Lipids). Abbreviations to lipid subclasses are described in Supp. Table S-4. (xlsx)

Supp. Table S-3. Lipid identifications for tiers 1 (MS/MS spectral match with precursor $\mathrm{m} / \mathrm{z}$ error $\leq 5.0 \mathrm{mDa}, \mathrm{mSigma} \leq 150$ and $\mathrm{MS} / \mathrm{MS}$ score $\geq 500), 2$ (MS/MS spectral match with precursor $\mathrm{m} / \mathrm{z}$ error $\leq 5.0 \mathrm{mDa}, \mathrm{mSigma} \leq 50$ and $\mathrm{MS} / \mathrm{MS}$ score $\geq 100$ ) and 3 (putative mass match with $\mathrm{m} / \mathrm{z}$ error $\leq 5.0 \mathrm{mDa}$ ). Abbreviations to lipid classes and subclasses are described in Supp. Table S-4. (xlsx)

Supp. Table S-4. Abbreviations for lipid classes and subclasses. ( $x l s x)$

Supp. Table S-5. Volcano plot analysis for cystic fibrosis patients (CF) compared to healthy controls. $(x l s x)$

Supp. Table S-6. Non-parametric ANOVA results for CF genotypic classes II, III, IV, V and VI. $(x l s x)$

Supp. Table S-7. Volcano plot analysis for cystic fibrosis patients with class III and IV mutations. $(x l s x)$

Supp. Table S-8. Volcano plot analysis for cystic fibrosis patients with class VI and V mutations. $(x l s x)$

Supp. Table S-9. Volcano plot analysis for cystic fibrosis patients with class III and V mutations. $(x l s x)$

Supp. Table S-10. Volcano plot analysis for cystic fibrosis patients with predicted FEV1\% above and below the median of $47.5 \%$. ( $x l s x)$ 
Supp. Table S-11. Volcano plot analysis for the cystic fibrosis patients above and below the age median of 20 years old. $(x l s x)$

Supp. Table S-12. Volcano plot analysis for the female and male cystic fibrosis patients. (xlsx)

Supp. Figure S-1. Representative base peak chromatograms (BPC) obtained under positive (A, $\mathrm{C}, \mathrm{E}, \mathrm{G}$ and $\mathrm{I})$ and negative (B, D, F, H and J) ionization for (A) and (B) a randomly chosen control sample; (C) and (D) a randomly chosen CF sample; (E) and (F) quality control (QC) injections; (G) and (H) a blank extract, i.e., extraction of water instead of the sample; and (I) and (J) the internal standard mixture, composed by 14 deuterated lipids (Supp. Table S-2). (pptx)

Supp. Figure S-2. PCA score plot for healthy controls (red) compared to cystic fibrosis patients with quality control (QC) extraction and injection replicates. (pptx)

Supp. Figure S-3. Boxplots for significantly altered lipids with the highest fold-changes (FC) for the comparison between cystic fibrosis patients (CF) and healthy controls identified in tiers 1 or 2 (MS/MS identification). (pptx)

Supp. Figure S-4. Boxplots for significantly altered lipids with the highest fold-changes (FC) for the comparison between cystic fibrosis patients $(\mathrm{CF})$ and healthy controls identified in tier 3 (putative mass-match identification). (pptx)

Supp. Figure S-5. Significantly altered lipids (fold change, FC $\geq 1.5$ or $\leq 0.67$ and p-value adjusted for false-discovery rate, $\mathrm{p}<0.05)$ for the comparison between cystic fibrosis patients (CF) and healthy controls, divided into $\mathrm{FC}(\mathrm{CF} /$ control) $\geq 1.5$ (blue) or $\leq 0.67$ (orange). Lipids with FC $\geq 1.5$ displayed higher normalized intensities for the CF patients, while lipids with FC $\leq 0.67$ had lower normalized intensities when compared to the healthy controls. (pptx) 
Supp. Figure S-6. All lipids employed for Receiver Operating Characteristic (ROC) analysis (blue) compared to lipids that resulted in area-under-the-curve of 1 (orange), divided by lipid subclasses, for the healthy controls and CF patients. (pptx)

Supp. Figure S-7. Extracted ion chromatograms obtained for two distinct biomarker candidates selected for differentiating cystic fibrosis patients from healthy controls, namely P373.25848/1.86 and P385.2584/2.01. (pptx)

Supp. Figure S-8. Extracted ion chromatograms obtained for two distinct biomarker candidates selected for differentiating cystic fibrosis patients from healthy controls, namely P784.58482/8.2 and 784.584841/9.16, identified as PC 15:1_21:2(tier 1). (pptx)

Supp. Figure S-9. Extracted ion chromatograms obtained for two distinct biomarker candidates selected for differentiating cystic fibrosis patients from healthy controls, namely P852.61033/9.81 and P495.34425/1.25. (pptx) 
Positive ionization

(A) Control sample

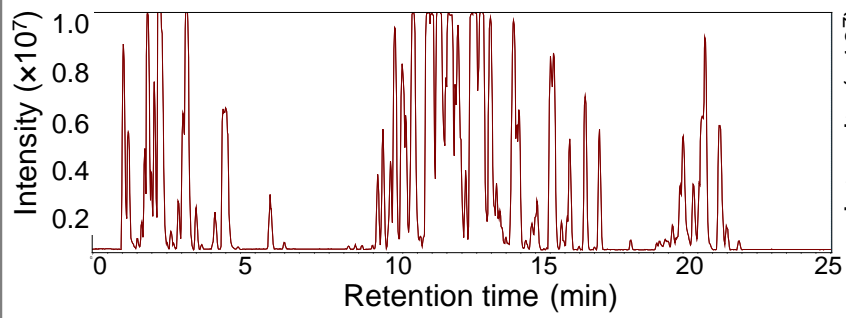

(C) CF sample

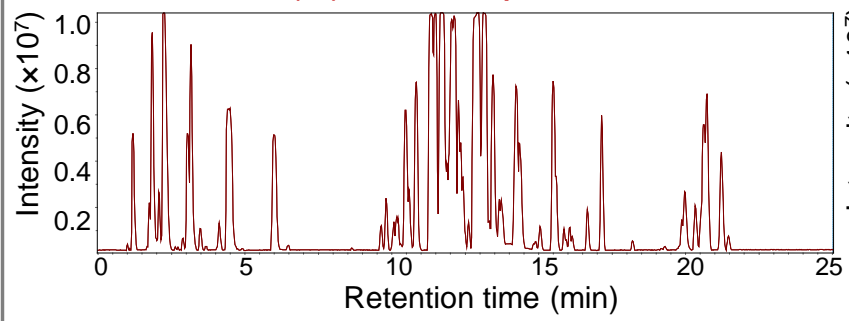

(E) QC injection

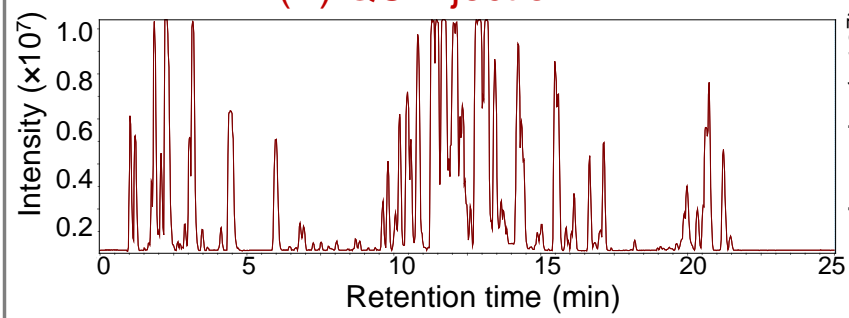

(G) Blank extract

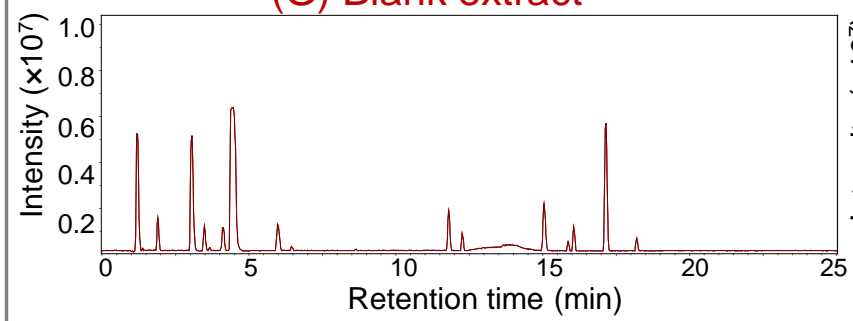

(I) Internal standards

\section{瓜}
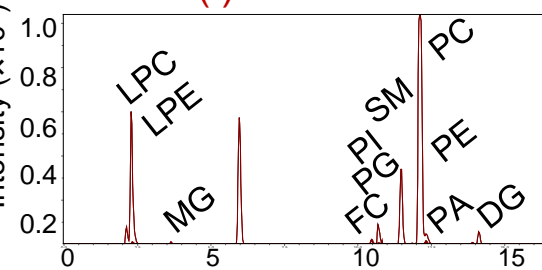

Retention time (min)
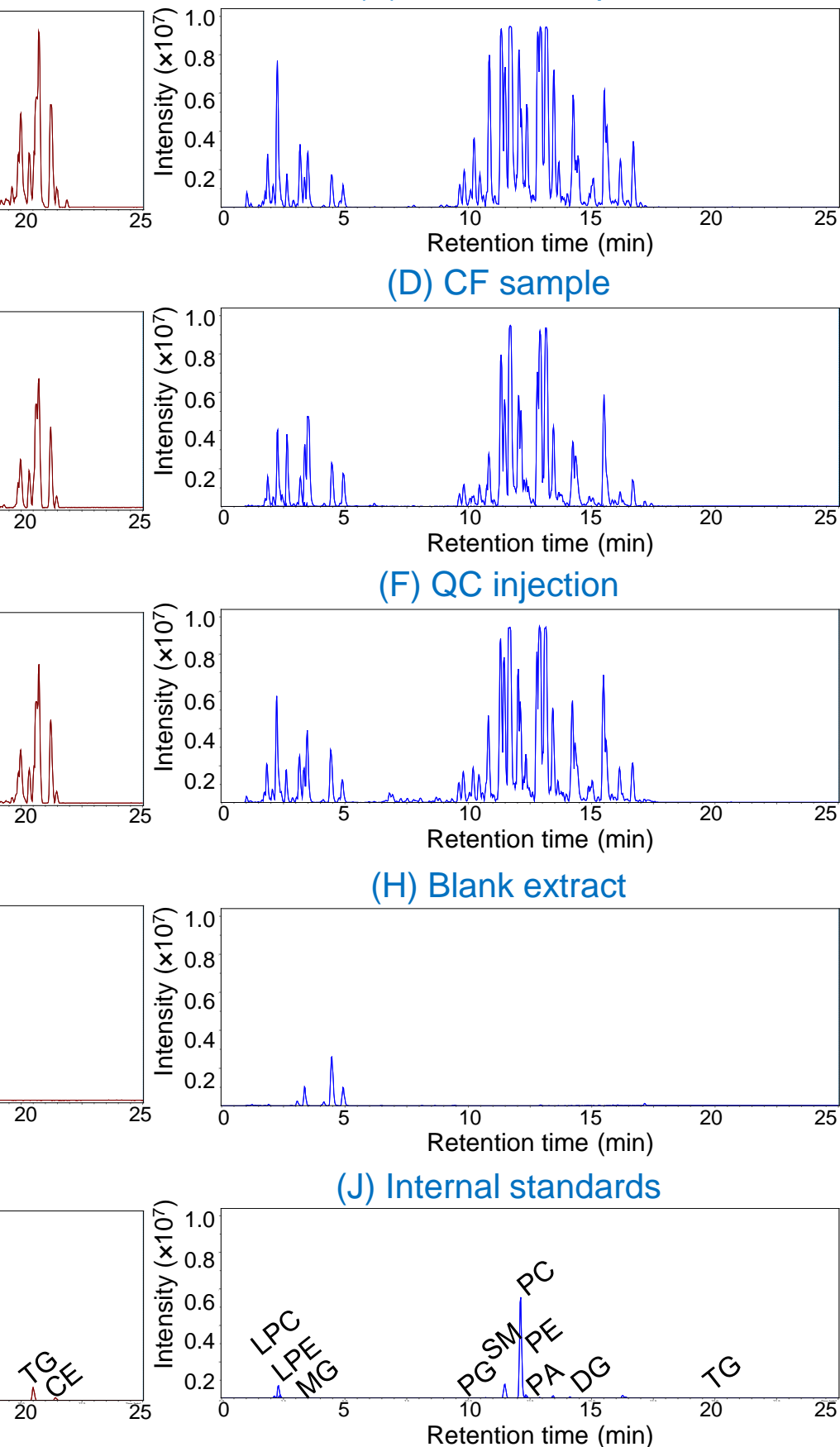

(D) CF sample

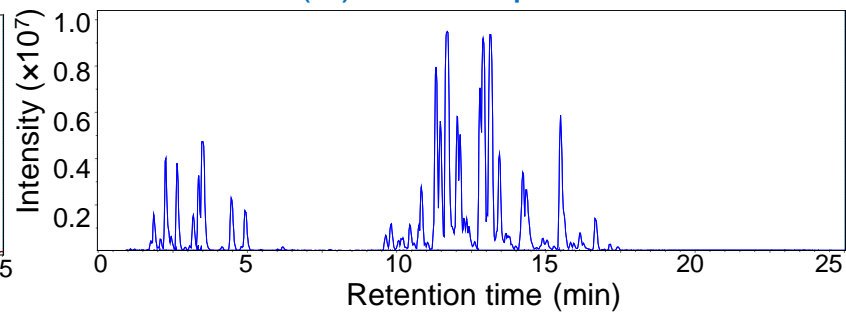

(F) QC injection

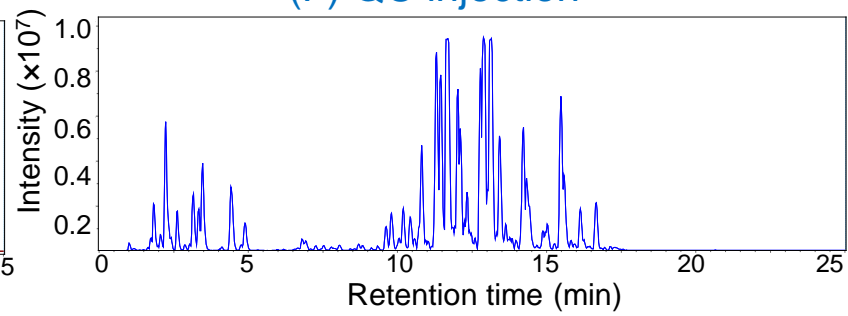

(H) Blank extract

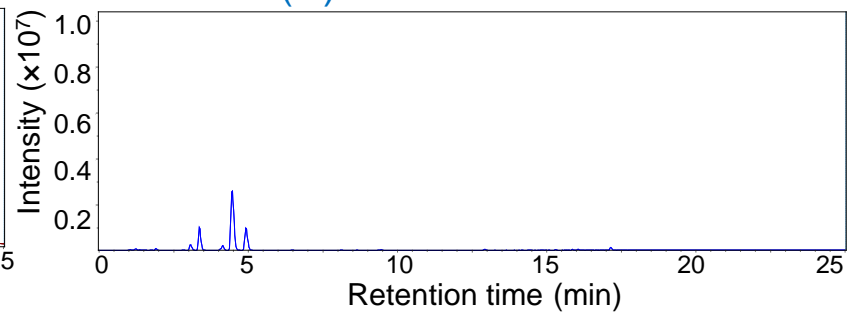

(J) Internal standards

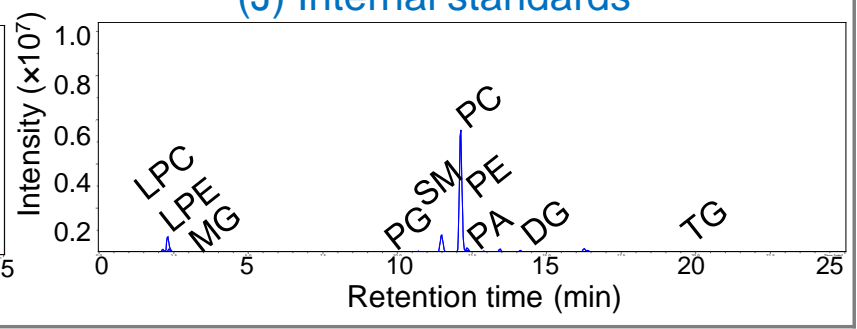

Supp. Figure S1. Representative base peak chromatograms obtained under positive (A, C, E, G and I) and negative (B,D, F, H and J) for: (A) and (B) a randomly chosen control sample; (C) and (D) a randomly chosen CF sample; (E) and (F) quality control (QC) injections; (G) and (H) a blank extract, i.e., extraction of water instead of sample; and (I) and (J) the internal standard mixture, composed by 14 deuterated lipids (Supp. Table S2). 


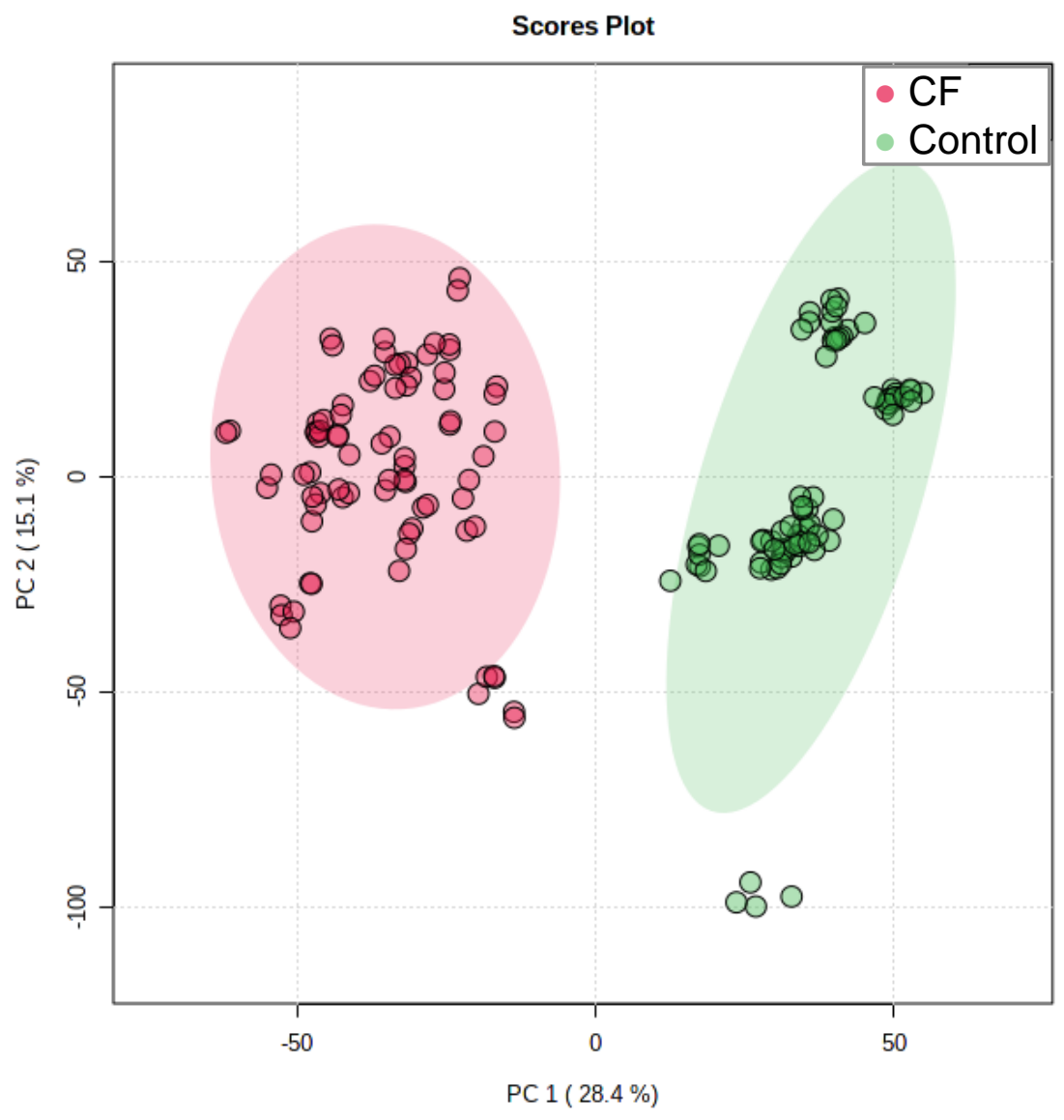

Supp. Figure S2. PCA score plot for healthy controls (red) compared to cystic fibrosis patients with quality control (QC) extraction and injection replicates. 
P784.58482/8.5

PC 15:1/21:2

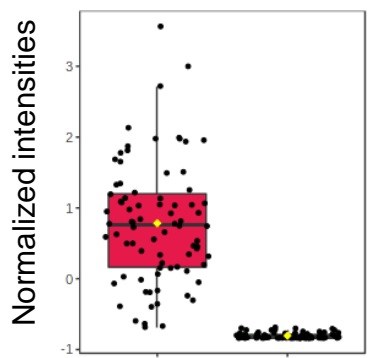

U
P810.600481/8.6

PC 19:2/19:2

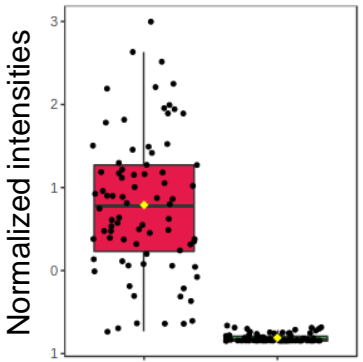

U

P942.81009/18.65 DGTS 25:0/24:4
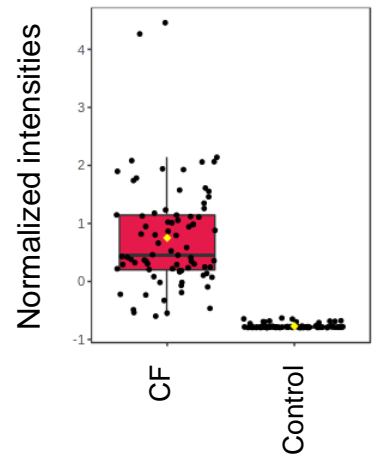

P810.60048/9.02 PC 38:4
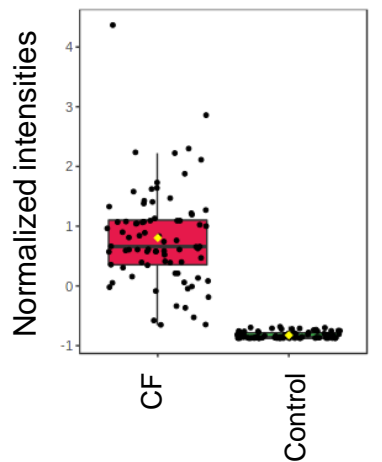

P784.584841/9.16

PC 15:1/21:2

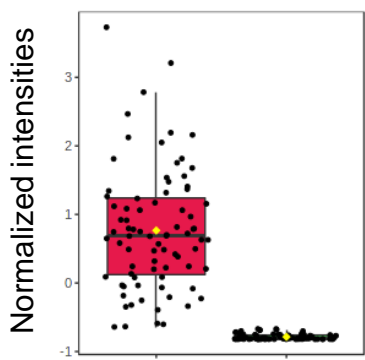

U

$\overline{0}$
등

N846.58547/8.74 PC 36:1;0; cyclo

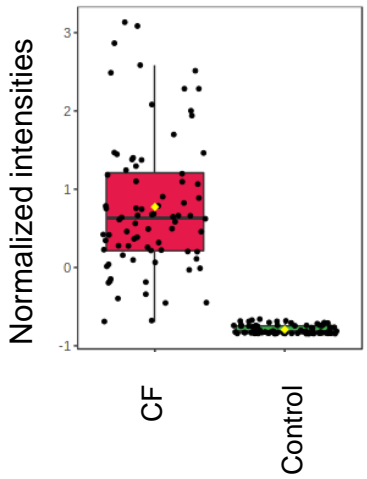

N842.55419/8.09 PC 36:3;0;cyclo

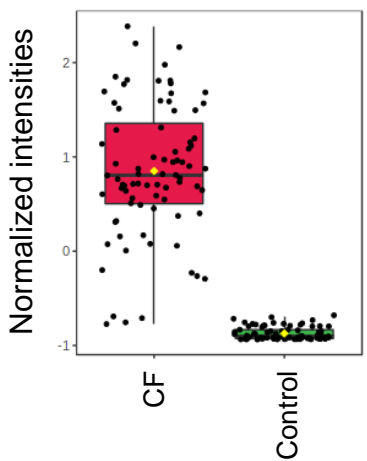

N828.57477/8.41

PS 22:0/17:2

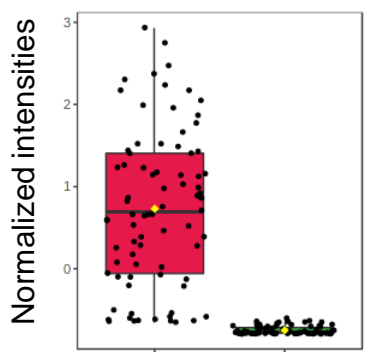

u

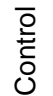

N854.59051/8.72 PS 19:0/22:3

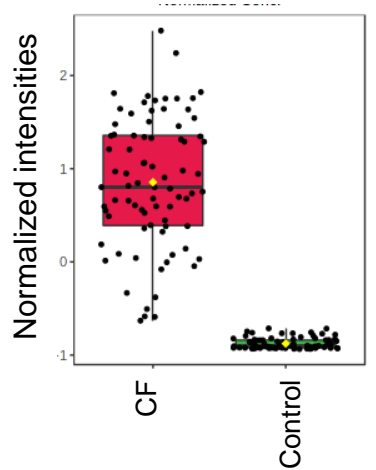

P784.58365/8.1 PC 14:1/22:2

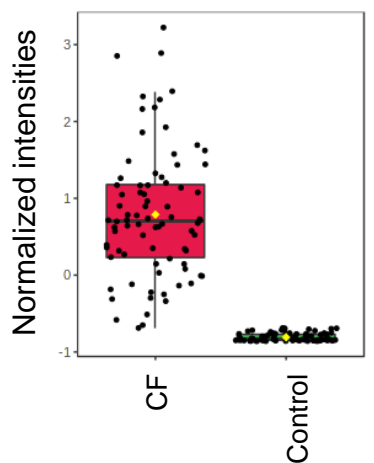

Supp. Figure S3. Boxplots for significantly altered lipids with the highest fold-changes (FC) for the comparison between cystic fibrosis patients (CF) and healthy controls, identified in tiers 1 or 2 (MS/MS positive identification). 


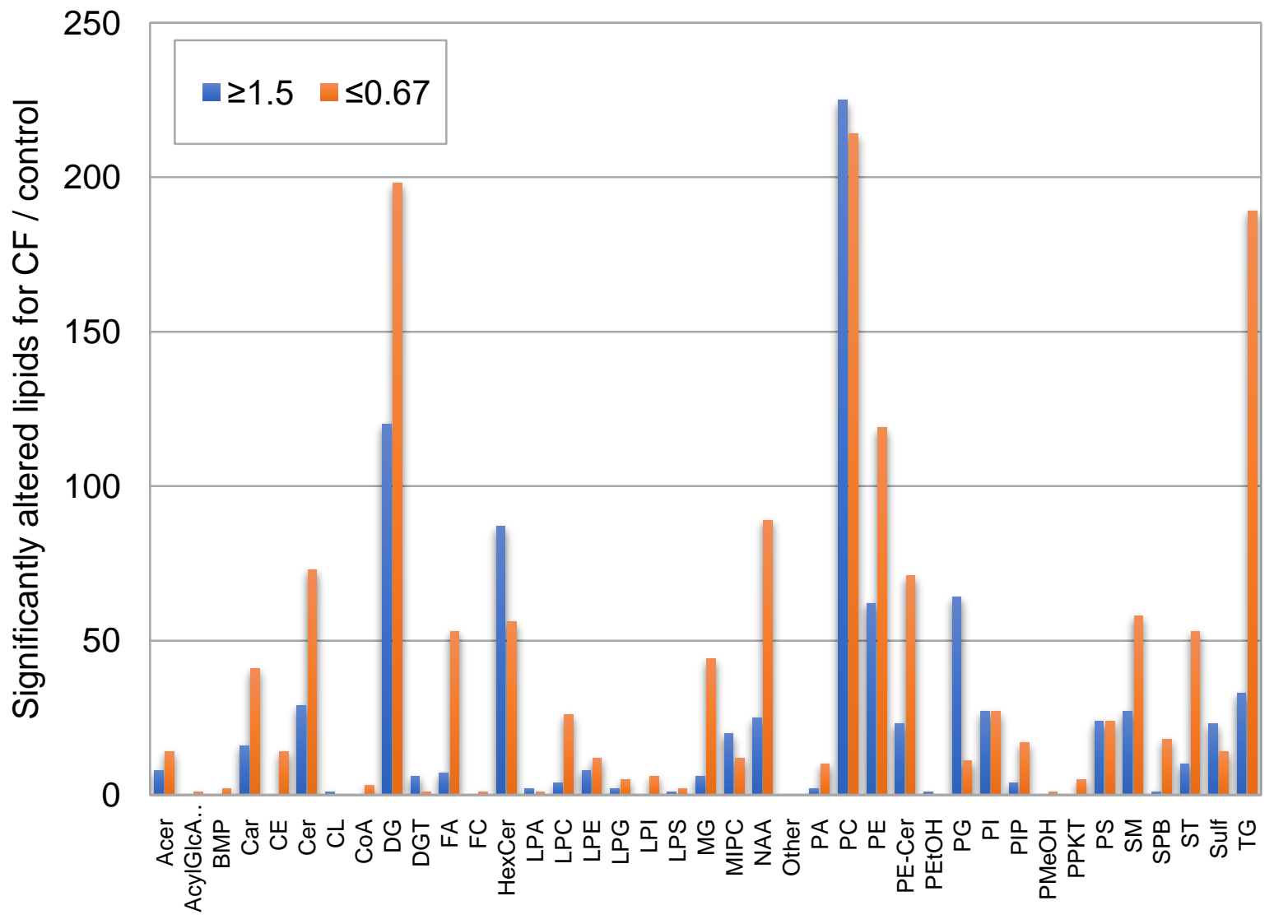

Supp. Figure S5. Significantly altered lipids (fold change, FC $\geq 1.5$ or $\leq 0.67$ and p-value adjusted for false-discovery rate, $\mathrm{p}<0.05$ ) for the comparison between cystic fibrosis patients (CF) and healthy controls, divided into FC (CF / control) $\geq 1.5$ (blue) or $\leq 0.67$ (orange). Lipids with FC $\geq 1.5$ displayed higher normalized intensities for the CF patients, while lipids with FC $\leq 0.67$ had lower normalized intensities when compared to the healthy controls. 


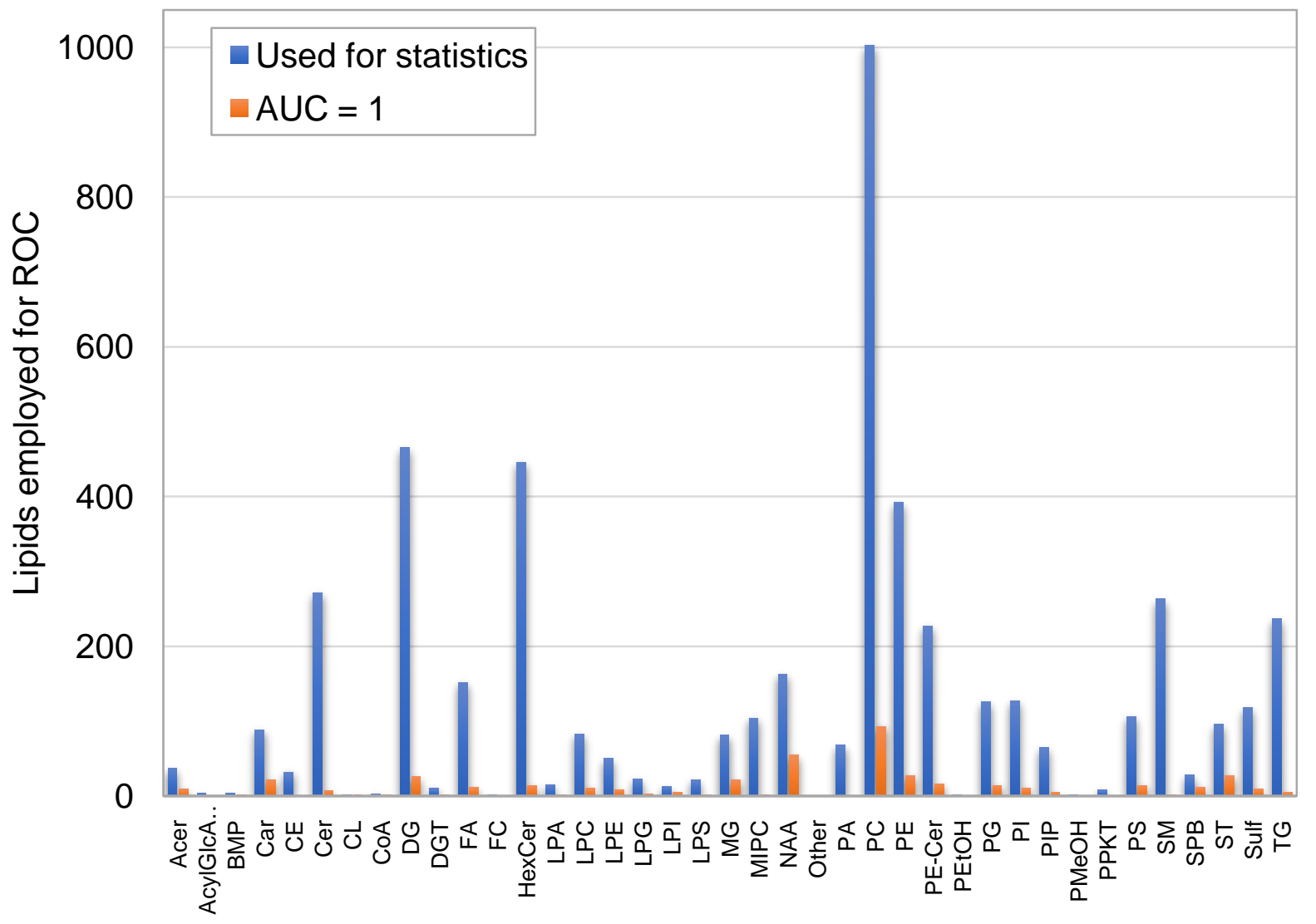

Supp. Figure S6. All lipids employed for Receiver Operating Characteristic (ROC) analysis (blue) compared to lipids that resulted in area-under-the-curve of 1 (orange), divided by lipid subclasses, for the healthy controls and CF patients. 

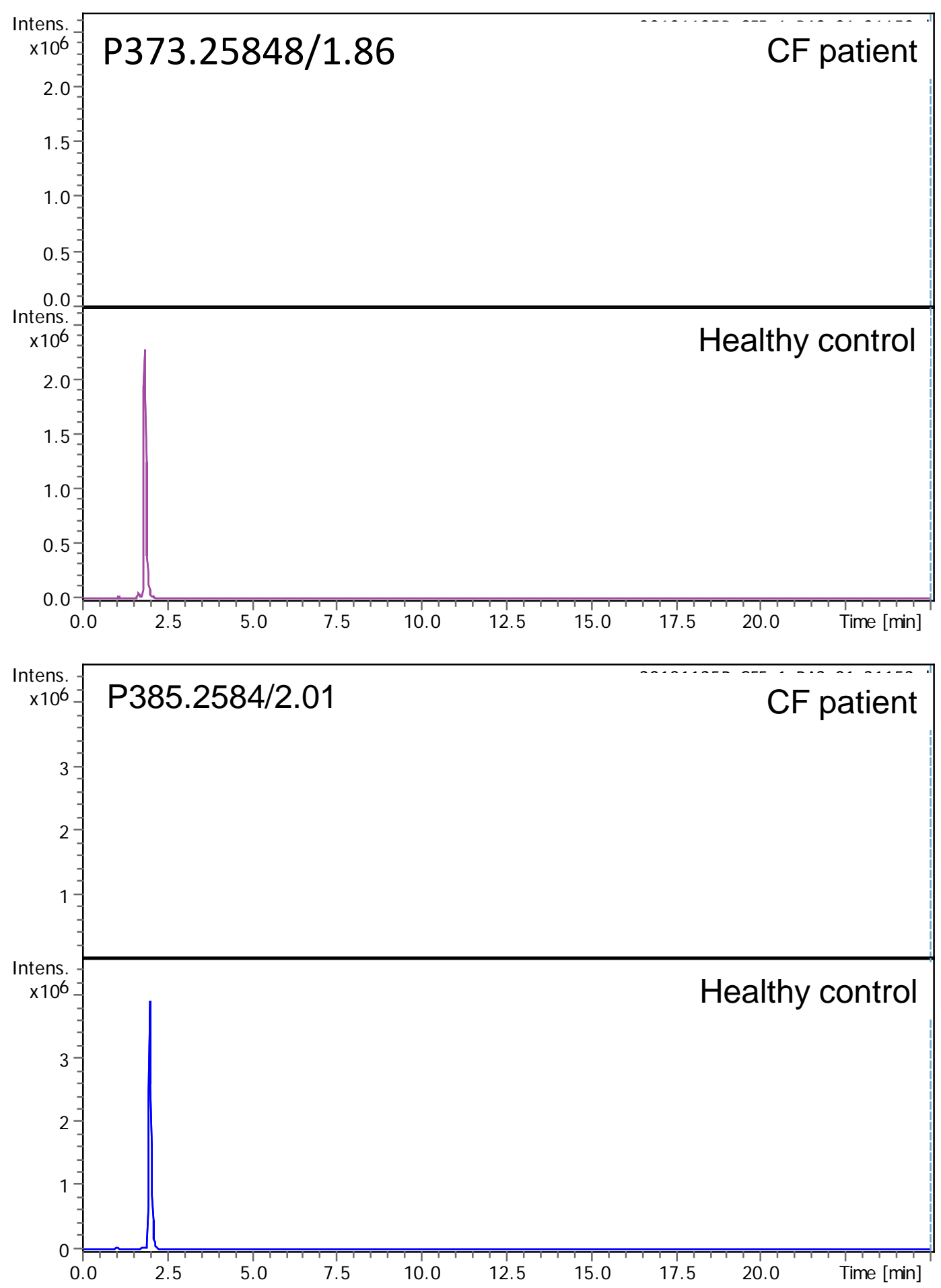

Supp. Figure S7. Extracted ion chromatograms obtained for two distinct biomarker candidates selected for differentiating cystic fibrosis patients from healthy controls, namely P373.25848/1.86 and P385.2584/2.01. 


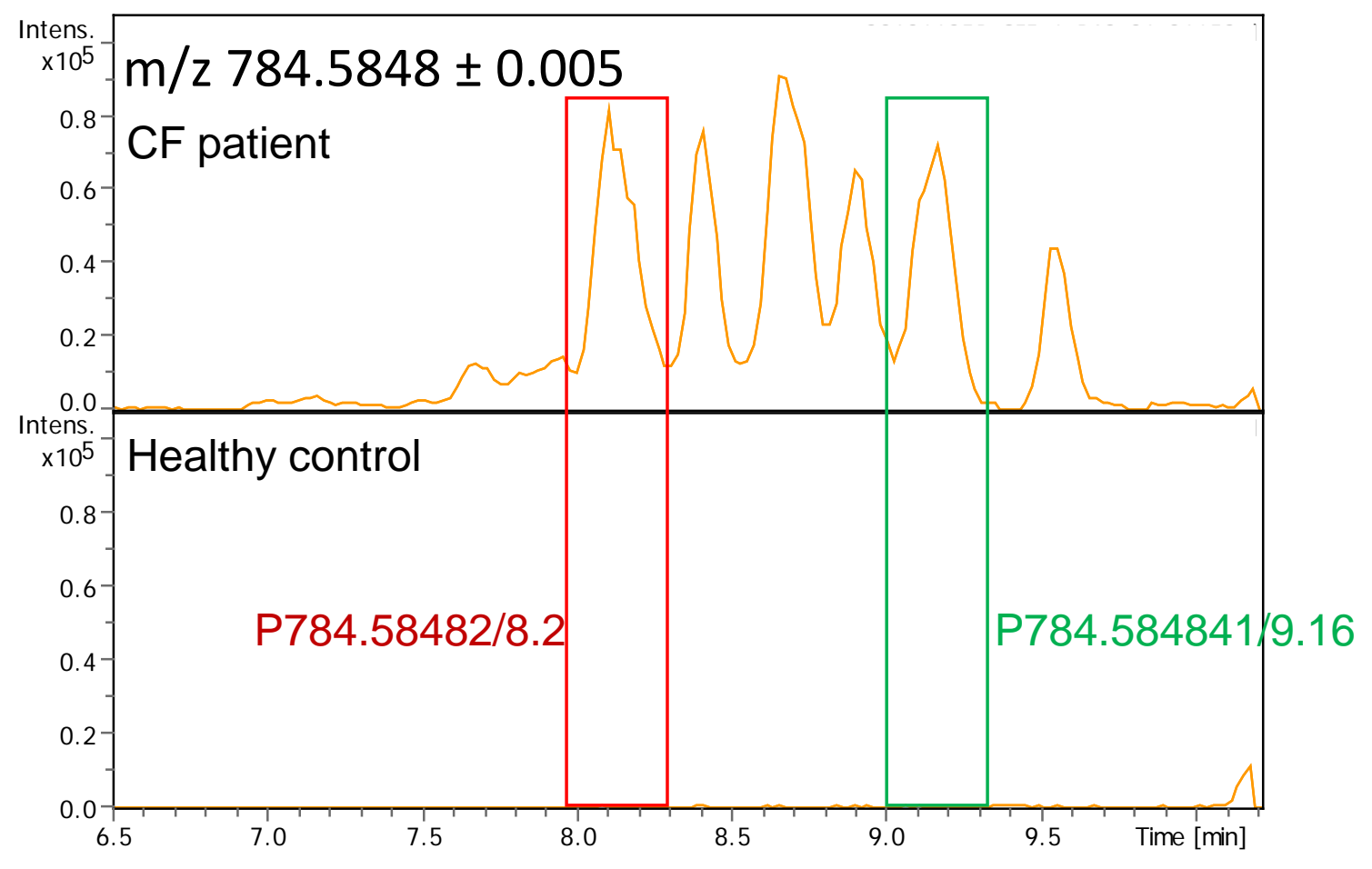

Supp. Figure S8. Extracted ion chromatograms obtained for two distinct biomarker candidates selected for differentiating cystic fibrosis patients from healthy controls, namely P784.58482/8.2 and 784.584841/9.16, identified as PC 15:1/21:2(tier 1). 

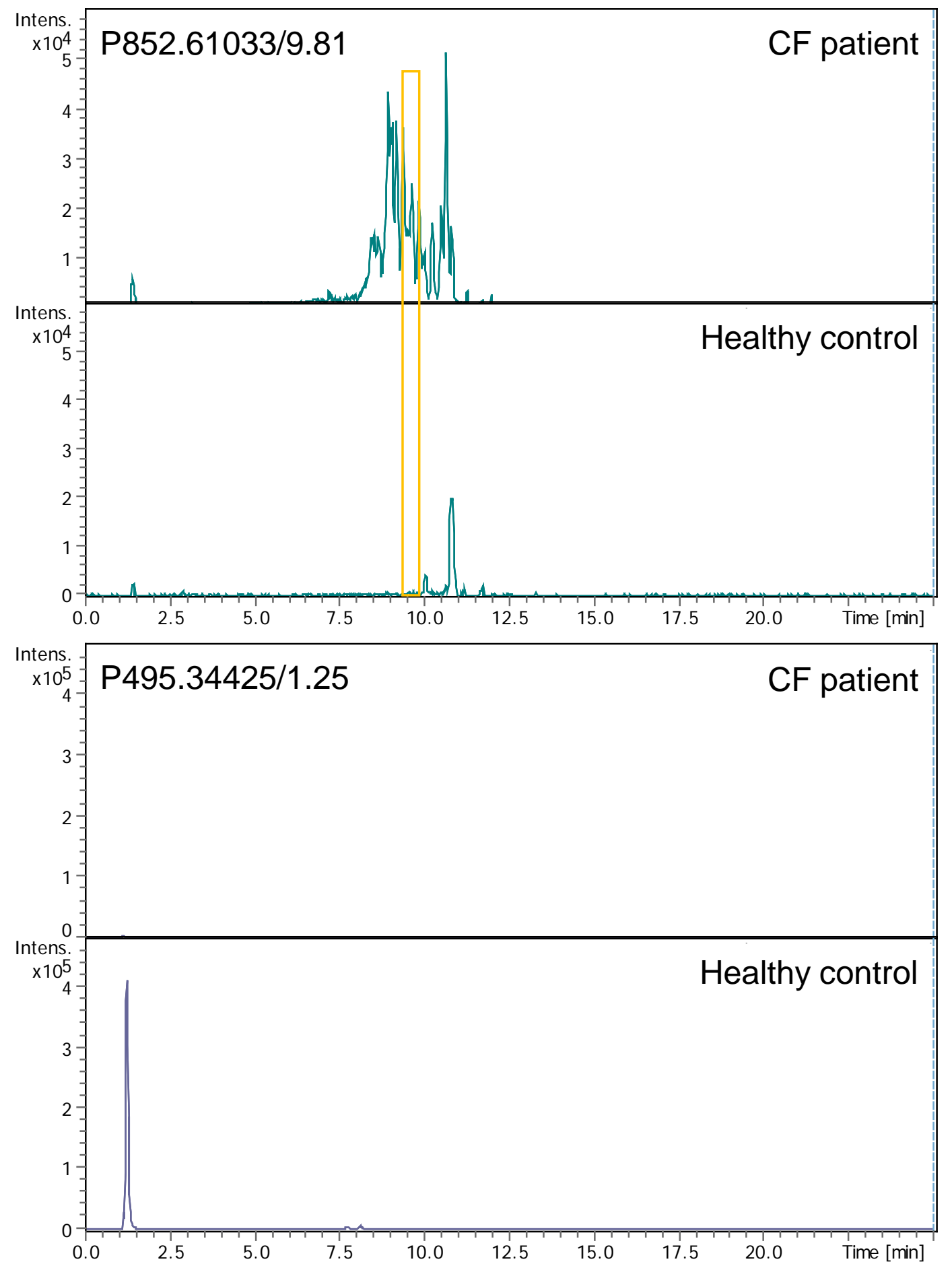

Supp. Figure S9. Extracted ion chromatograms obtained for two distinct biomarker candidates selected for differentiating cystic fibrosis patients from healthy controls, namely P373.25848/1.86 and P385.2584/2.01. 